Anno 34, 2019 / Fascicolo 1 / p. 148-150 - www.rivista-incontri.nl - http://doi.org/10.18352/incontri.10297 (c) The author(s) - Content is licensed under a Creative Commons Attribution 3.0 Unported License Publisher: Werkgroep Italië Studies, supported by Utrecht University Library Open Access Journals

\title{
Una panoramica dell'Italianistica iberica e latinoamericana
}

\author{
Recensione di: Alejandro Patat (a cura di), La letteratura italiana \\ nel mondo iberico e latinoamericano. Critica, traduzione, \\ istituzioni, Pisa, Pacini, 2018, 320 p., ISBN: 9788869954559, \\ $€ 20,00$.
}

\section{Nicolás M. Sánchez Gasparini}

Se messi a confronto con gli altri centri di studi italiani sparsi per il mondo, quelli presenti nell'America latina e nella penisola iberica risultano aver raggiunto una posizione notevole su numerosi fronti di ricerca che vanno al di là del comparativismo. Questo volume ne dà prova attraverso una selezione rappresentativa degli studi correnti in materia di ricezione, traduzione, critica e insegnamento. Composto soprattutto da contributi che sono stati presentati al congresso 'La letteratura italiana nel mondo iberico e latinoamericano. Poli culturali, traduzione e critica', tenuto presso l'Università per Stranieri di Siena il 19 e 20 gennaio 2017, questo volume costituisce una lettura utile per chi voglia informarsi sulla situazione attuale della disciplina in due realtà specifiche, vale a dire il mondo portoghese e quello ispanofono, i quali storicamente intrattengono rapporti stretti e proficui con la cultura italiana.

Il lavoro è diviso in tre sezioni: 'Critica', 'Traduzione' e 'Istituzioni', precedute da una prefazione e un'introduzione.

Nella prefazione Alejandro Patat riassume e soppesa criticamente il contenuto degli articoli ospitati nel volume e conclude sottolineando il bisogno di sviluppare l'interazione fra i centri di studi italiani. Segue l'introduzione di Marcello Ciccuto che espone un caso paradigmatico dell'italianistica latinoamericana: l'analisi di Borges della Commedia.

La prima sezione del volume, 'Critica', getta uno sguardo sulle linee di ricerca oggi seguite nei centri di studi nazionali e internazionali. Apre la sezione un articolo su come un gruppo di studiosi dell'Università Complutense di Madrid, sotto la guida di Juan Ignacio Varela-Portas de Orduña, abbia proposto di ritornare all'interpretazione allegorica di Dante, malgrado l'atteggiamento interpretativo antiallegorico che ha caratterizzato l'esegesi dantesca fin dall'Ottocento. Lorenzo Bartoli, a sua volta, commenta un progetto teatrale realizzato dagli attori-detenuti del braccio di Alta Sicurezza del carcere di Rebibbia a Roma, nel quale l'Inferno di Dante viene rappresentato attraverso le esperienze e i dialetti dei reclusi - inclusa una varietà dello spagnolo americano. Il suo contributo si chiude con una riflessione sulla potenza catartica della letteratura, in opposizione alla logica retributiva della giustizia. Il 
lavoro di Marco Carmello, poi, indaga sul trattamento e sullo sviluppo dello spazio argentino nelle opere e nella poetica di Gadda. L'ipotesi soggiacente a tale contributo è che Gadda abbia compiuto un travestimento "alla sudamericana" dei luoghi della sua infanzia lombarda allo scopo di aprirsi uno spazio libero di scrittura. Almada Ordóñez, invece, commenta l'arrivo in Argentina dei futuristi Marinetti, Ungaretti e Puccini negli anni del post-futurismo. Facendo un uso cruciale dei nuovi documenti ottenuti nelle emeroteche argentine, Ordóñez aggiunge un nuovo tassello critico, interessato alla controversia politica e al proselitismo fascista assunto dai futuristi. Graciela Caram fa un'analisi dettagliata dell'influsso enorme dell'opera di Pavese sull'aggiornamento della letteratura argentina avvenuto negli anni Sessanta. Lo studioso giapponese Hideyuki Doi riporta i risultati della sua indagine concernente la figura imponente di Gherardo Marone, intellettuale italiano molto coinvolto nei circoli culturali napoletani tra gli anni Dieci e Venti, e fondatore degli studi d'Italianistica in Argentina. Il lavoro approfondito di Davide Toma, che chiude la sezione, mette a confronto le divergenti politiche linguistiche dell'italiano adottate in due paesi ispanofoni, la Spagna e l'Argentina.

La seconda sezione, 'Traduzione', si concentra sui problemi, vecchi e nuovi, che ruotano attorno al tema della traduzione della letteratura italiana. A capo di questa seconda sezione, Patricia Peterle, Andrea Santurbano e Lucia Wataghin presentano il loro vasto progetto di ricerca sulla traduzione della letteratura italiana in Brasile, ancora in corso. Il progetto, che rappresenta un punto di incontro tra traduzione letteraria e letteratura comparata, esplora le possibilità della traduzione come veicolo transculturale in un sistema letterario globale. Fernando Ibarra si concentra in modo simile sull'influenza esercitata dalla letteratura italiana sugli scrittori messicani nella prima metà dell'Ottocento, che avrebbero cercato di forgiare una nuova identità culturale fatta a misura di quella europea. Claudia Fernández Greco, dantista argentina, offre una sintesi della sua ricerca di dottorato percorrendo la storia della traduzione di Dante in Argentina, e giunge alla conclusione che la tradizione traduttiva della Commedia sia una pietra miliare della cultura argentina stessa. La laboriosa ricostruzione eseguita da Hernández González, invece, ci consegna i ritratti di Rafael Cansinos Assens e Ángel Crespo, scrittori-traduttori di letteratura italiana in Spagna. Questa ricostruzione ci invita a considerare, da un lato, quanto importante possa essere il ruolo del traduttore per la sorte dell'opera originale nel sistema culturale d'arrivo, e dall'altro, come la frequentazione degli autori tradotti agisca sull'opera stessa del traduttore. L'ultimo lavoro di questa sezione, firmato da Alejandro Patat, è dedicato alla discussione di vecchia data sulle differenze che oppongono la traduzione della letteratura italiana nello spagnolo peninsulare, a quella nelle lingue ispanoamericane, e si conclude con un approfondimento degli aspetti politici ed estetici della questione.

La terza sezione, 'Istituzioni', comprende una serie di interventi su istituti e figure particolarmente impegnati negli studi italiani in America latina e nella penisola iberica. Il lavoro di Carmen Blanco Valdés e Linda Garosi ci offre una panoramica dell'insegnamento della letteratura italiana nel sistema universitario spagnolo, e si conclude con una valutazione delle sue carenze. Gaspare Trapani, invece, descrive le carenze che caratterizzano l'insegnamento della letteratura italiana a Lisbona. Un rimedio a tali carenze potrebbe essere l'introduzione dell'italiano come lingua straniera nelle scuole portoghesi. Vicente González Martín, a sua volta, presenta un resoconto della tradizione storica che fa dell'ateneo di Salamanca uno dei più autorevoli centri di studi italiani. In aggiunta, Carlos Gatti e Jorge Wiesse espongono la storia della Cátedra de Lectura Dantis a Lima. La storia della Cattedra Straordinaria Italo Calvino presso l'Universidad Nacional Autónoma de México è riportata da Sabina Longhitano e Rodrigo Jardón, i quali presentano, inoltre, una panoramica della 
traduzione della letteratura italiana in Messico, che oggi si trova in un periodo di grande sviluppo. Il libro si conclude con un apporto prezioso di Nora Sforza, che ci consegna le biografie di due figure preminenti dell'Italianistica a Buenos Aires.

Il volume, in sintesi, costituisce uno strumento di consultazione di approfondimento bibliografico per chi, allargando i confini dell'Italianistica, ha curiosità di sapere che cosa si studi nel vastissimo continente del mondo ispanofono e lusofono.

Nicolás M. Sánchez Gasparini

Ciudad de la Paz 2879, 1 E

Buenos Aires C.P. 1428 (Argentina)

nsanchezgasparini@gmail.com 